\title{
CW Nd:YVO 4 disk laser with multipoint diode pumping and in-phase super-mode lasing
}

\author{
D. A. Guryev ${ }^{1}$, D. A. Nikolaev $^{1}$, V. B. Tsvetkov ${ }^{1}$ \\ 1. Prokhorov General Physics Institute, Russian Academy of Sciences, 38 Vavilov Str., 119991 Moscow, Russian Federation
}

Development of large aperture lasers with diffraction limited output is a relevant problem of laser physics. The use of the degenerated type cavity is a good way to solve this problem. In the degenerated cavity all pump areas can take part in the in-phase collective lasing of a diffraction limited output beam. In present work we demonstrate in-phase super-mode lasing regime in the solid-state disk laser with multipoint diode pumping and results of research of its lasing conditions.

The laser cavity was formed by a flat HR mirror placed on the back surface of an active disk (AD) and a flat output mirror (both coated at $\lambda=1064$ lasing wavelengths): The antireflection coating has been covered on forward surface of the AD for the pump $(808 \mathrm{~nm})$ and lasing wavelengths. the $\mathrm{AD}$ was a $20 \times 20 \times 0.5 \mathrm{~mm}^{3}$ $\mathrm{Nd}: \mathrm{YVO}_{4}$ crystal. The continious-wave laser diode was used to pump the AD. Pump radiation inside the AD was a one-dimensional spatially-periodic pumping points pattern and consisted of 9 spots with the center distance $\mathrm{d}=0.5 \mathrm{~mm}$.

Transverse distributions of laser radiation in a far-field zone and inside the $\mathrm{AD}$ were researched in dependence on absorbed pump power $\mathrm{P}_{\text {in }}(0-4 \mathrm{~W})$ and cavity length $\mathrm{L}(6-19 \mathrm{~cm})$.

The results of the research showed that laser output had a spatial-periodic structure both in a far-field zone and inside the $\mathrm{AD}$ (Figure 1 (a) and (b) respectively) in a range of cavity length values $\mathrm{L}=6-11 \mathrm{~cm}$ with all pump power levels and in a range of cavity length values $\mathrm{L}=12-19 \mathrm{~cm}$ with absorbed pump power lower than $3 \mathrm{~W}$. Maximum output power was $250 \mathrm{~mW}$. A similar picture also had been observed and explained in paper [1]. In the field of cavity lengths $\mathrm{L}=12-19 \mathrm{~cm}$ and $\mathrm{P}_{\mathrm{in}}=3-4 \mathrm{~W}$ the lasing appeared at $\mathrm{N}=5-7$ neighboring pumping points. The transverse intensity distribution of the laser emission inside the AD (Figure 1 (c)) did not possess the spatially periodic character in contrast with the results for another area. The angular structure of radiation in a far-field zone represented an intensive central peak and two weak petals (Figure 1 (d)). The output power in the central lobe reached $80 \%$ from the total lasing output, and its full divergence $2 \mathrm{~V}$ along axis $\mathrm{Y}$ made $2 \mathrm{~V}=\lambda /\left(\mathrm{N}^{*} \mathrm{~d}\right)$. Thus, in this range of values $\mathrm{P}_{\text {in }}$ and $\mathrm{L}$ the in-phase supermode lasing was observed. Output power reached 180 $\mathrm{mW}$.

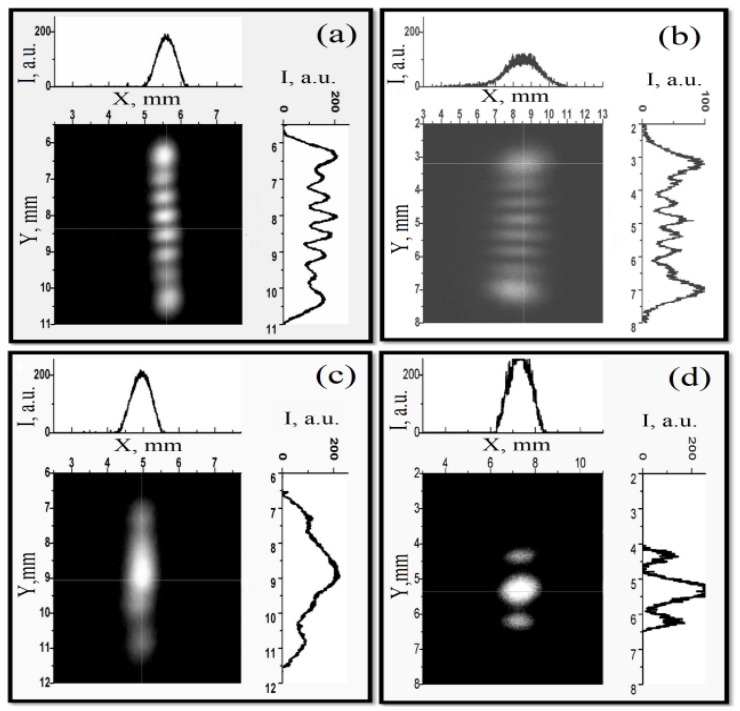

Fig. 1 The radiation intensity transverse distribution: (a) and (c) inside the AD in the condition of collective mode lasing and the super-mode, respectively; (b) and (d) in the far-field zone in the mode of collective mode lasing and in-phase supermode, respectively.

For an explanation of existence of a supermode lasing the study of the lens thermoinduced in the AD has been done. It has been shown that the supermode lasing may be realized when the laser cavity corresponded to parameters of a degenerate resonator. Based on this explanation the existence conditions of a supermode lasing have been defined for a wide range of pump power values.

\section{References}

[1]D. A. Guryev, D. A.Nikolaev, V. B. Tsvetkov, "Nd:GGG disk laser with multipoint spatially periodic optical pumping", Laser Phys. Lett. 13, 045003 (2016). 